\title{
«Der 1. April soll den Beginn einer neuen Ära markieren»
}

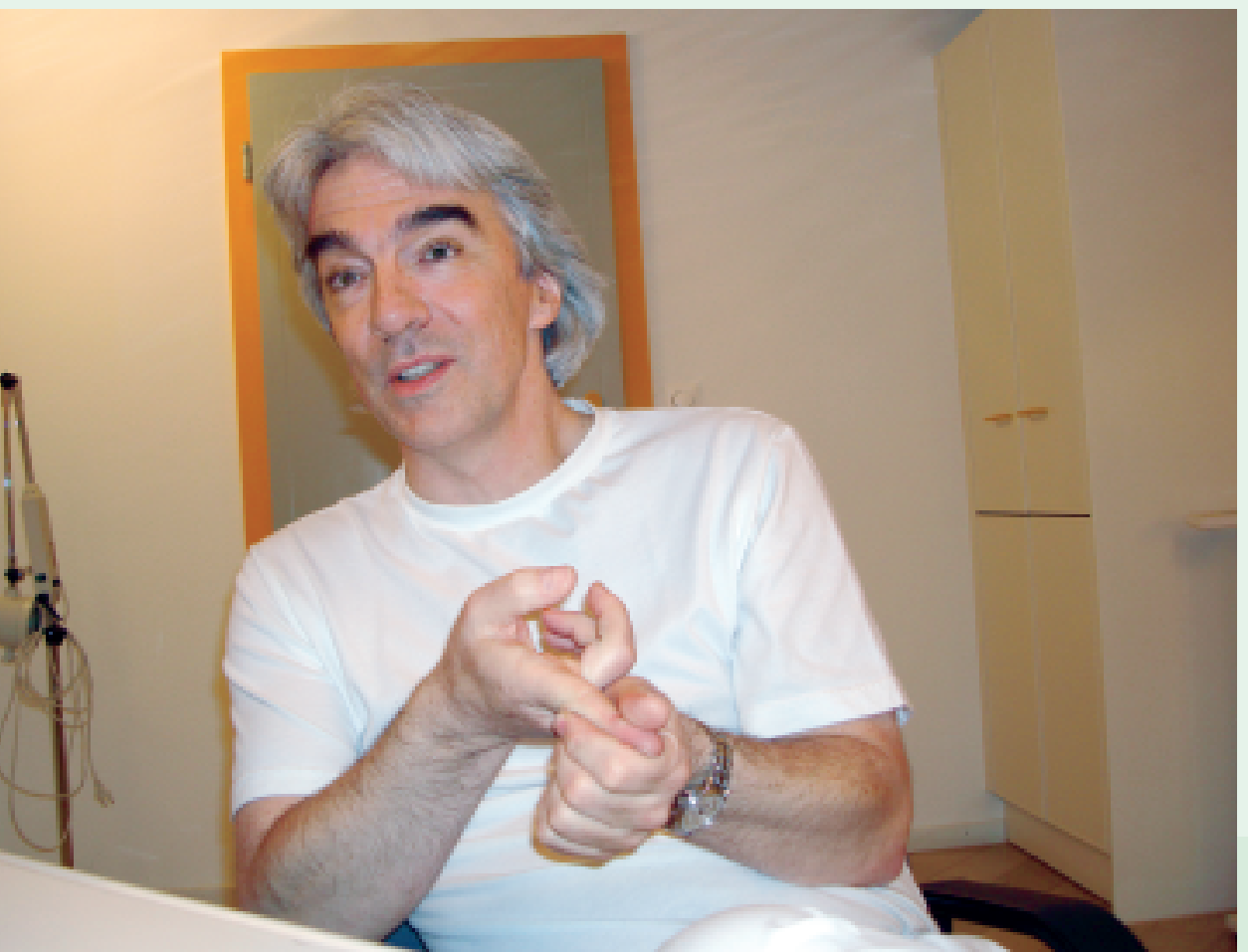

Interview:

Bruno Kesseli
SÄZ: Die Ärzte auf der Strasse, das ist etwa so wie Schnee in der Sahara. Erklären Sie uns doch bitte, was passiert ist.

Dr. med. Hansueli Späth, Präsident SGAM: Es ist schlicht zuviel passiert. Die Bombe ist einfach geplatzt, ganz kurz gesagt. Natürlich ist die «Demo» die Folge einer längeren Entwicklung, aber zuletzt hat auch der Zufall eine Rolle gespielt. Und zwar insofern, als die bundesrätlichen Entscheidungen von Herrn Couchepin bezüglich Generika, bezüglich MiGel und vor allem bezüglich Analysenlistentarif zum Zeitpunkt unserer SGAM-Jahresversammlung in Luzern publik

\section{«Wir werden sicher ein Institut oder einen Lehrstuhl für Hausarztmedizin an jeder Universität mit einer medizinischen Fakultät verlangen.»}

Die Spannung steigt: In 10 Tagen werden die Grundversorger und diejenigen, die sie unterstützen, ab $14 \mathrm{Uhr}$ auf dem Bundesplatz für ihre Anliegen demonstrieren. Wir befragten Hansueli Späth, Präsident der SGAM und einer der Initiatoren der «Demo», zur Bedeutung dieses Anlasses für die Hausarztmedizin und die Ärzteschaft insgesamt.

Hofft auf die Solidarität der gesamten Ärzteschaft: SGAM-Präsident Hansueli Späth im Gespräch.

wurden. Die Empörung unter den Kollegen war riesig. Sie war so gross, dass es nicht einfach war, die verschiedenen Ideen für Aktionen zu mässigen. Da waren Kolleginnen und Kollegen, die sofort streiken wollten, es war von «Schandbriefen» die Rede, Herr Couchepin sollte per Post mit Laborröhrchen überschwemmt werden, es wurde angeregt, die Praxislabors für eine Woche zu schliessen und anderes mehr.

Sie entschieden sich schliesslich für eine Demonstration, um auf die Anliegen der Grundversorger aufmerksam zu machen. Welche Überlegungen waren dafür ausschlaggebend?

Viele der genannten Ideen hätten sich nicht mit genügend Breitenwirkung und medialer Resonanz realisieren lassen. Ein Streik beispielsweise macht nur dann Sinn, wenn mindestens drei Viertel oder noch besser 90 Prozent aller Betroffenen mitmachen, sonst bleibt eine solche Aktion wirkungslos. Es wäre schwierig gewesen, unsere Kollegen, die solche «Kampfmassnahmen» nicht gewohnt sind, für so etwas zu mobilisieren. 
Dagegen hat eine Kundgebung an einem Samstagnachmittag eine relativ tiefe Reizschwelle. Zeitlich lässt sich das einrichten, und man exponiert sich als Einzelperson nicht allzu stark. Vielmehr steht die Solidarisierung mit einer Gruppe von Kolleginnen und Kollegen im Vordergrund. Zudem kann mit einer grossen Demonstration via Medien einem breiten Publikum vermittelt werden, dass die Lage für uns Hausärzte schwierig ist, dass wir aber auch bereit sind, etwas dagegen zu unternehmen.

Die SGAM will die Kernanliegen der Grundversorger am Vortag der Demonstration an einer Pressekonferenz kommunizieren. Können Sie schon ein paar Anhaltspunkte geben, in welche Richtung ihre Forderungen gehen?

Es gibt zwei grosse Problemkreise: Die in den letzten Jahren kontinuierliche Schwächung der Hausarztmedizin und der drohende, teilweise sogar schon sehr manifeste Ärztemangel. Ein wichtiger Grund für die negativen Entwicklungen sind die offensichtlichen Defizite in der Aus- und Weiterbildung zum Hausarzt. In diesem Bereich wird ein Teil unserer Kernforderungen angesiedelt sein. Wir werden sicher ein Institut oder einen Lehrstuhl für Hausarztmedizin an jeder Universität mit einer medizinischen Fakultät verlangen. Immerhin ist die Hausarztmedizin gemäss WONCA* eine eigenständige akademische Disziplin. Ebenso sicher werden Associations of General Practitioners/Family Physicians. Kurzform: World Organization of Family Doctors wir die politische und finanzielle Unterstützung der Praxisassistenz verlangen, die in der Weiterbildung zum Hausarzt zentral ist.

Wenn man die Karriereoptionen für Ärzte unter finanziellen Gesichtspunkten betrachtet, ist die Hausarztmedizin ebenfalls nicht sehr attraktiv. Sicher ist es politisch heikel, das zu sagen...

Tatsächlich sind die finanziellen Bedingungen in diesem Segment im Vergleich zu anderen Fachrichtungen nicht eben berauschend. Ich sage das zwar nicht gern, es spielt aber eine nicht zu

\section{«Die Politiker werden nicht müde}

\section{zu betonen, wie wichtig die}

\section{Hausarztmedizin sei. Das sind} Lippenbekenntnisse. Die Realität

\section{sieht genau umgekehrt aus.»}

unterschätzende Rolle. Die fehlende Attraktivität für die jungen Kollegen hängt aber auch mit weiteren Faktoren zusammen, zum Beispiel mit der nach wie vor sehr hohen Präsenzzeit und den Notfalldiensten. Diesbezüglich erwarten wir Unterstützung durch das EDI und das BAG, beispielsweise durch tarifarische Massnahmen, die einen gewissen Ausgleich schaffen könnten. Der Hausarztberuf soll wieder attraktiver werden.

Fühlen sich die Grundversorger als Prügelknaben? Wenn man sich umhört oder sich die Leserbriefe in den Fachzeitschriften - auch in der Ärztezeitung - anschaut, bekommt man fast zwangsläufig diesen Eindruck. Es sind offensichtlich sehr viele negative Emotionen, viel Wut und Frust vorhanden.

Ja, das dürfte der Hauptgrund sein, weshalb die Demonstration hoffentlich ein Erfolg wird. Es ist offensichtlich, dass die Wut und die Empörung unter den Hausärzten nicht mehr aufzuhalten sind. Ich denke aber nicht, dass sich diese Wut gegen die «Spezialisten» richtet, auch wenn man uns dies immer wieder einreden will. Auch von politischer Seite heisst es immer wieder, schaut doch einmal zuerst unter euch. Ihr müsst in der FMH aufräumen. Der Kuchen muss umverteilt werden. Das ist aber keineswegs Sinn und Zweck unserer Aktion. Es geht nicht darum, dass die anderen irgendetwas abgeben müssen.

\section{Sondern?}

Wir fühlen uns in erster Linie als Prügelknaben der Ämter und Behörden. Die Politiker werden nicht müde zu betonen, wie wichtig die Haus- 
arztmedizin sei. Das sind Lippenbekenntnisse. Die Realität sieht genau umgekehrt aus. Wenn im Gesundheitswesen gespart werden muss, dann schaut man zuerst mal, was man bei den Hausärzten machen kann. Ob es Sinn macht, die Leberlebendtransplantationen via Tarmed zu bezahlen, das fragt kein Mensch. Aber niemand hat ein Problem damit, uns zweieinhalb Minuten der Konsultation abzuzwacken und zu sagen, damit spare man Millionen. Das muss jetzt aufhören. Ich erhoffe mir von der Demo unter anderem, dass das niemand mehr wagt. Tatsache ist doch, dass uns die Medizin ohne die Grundversorger aus dem Ruder läuft.

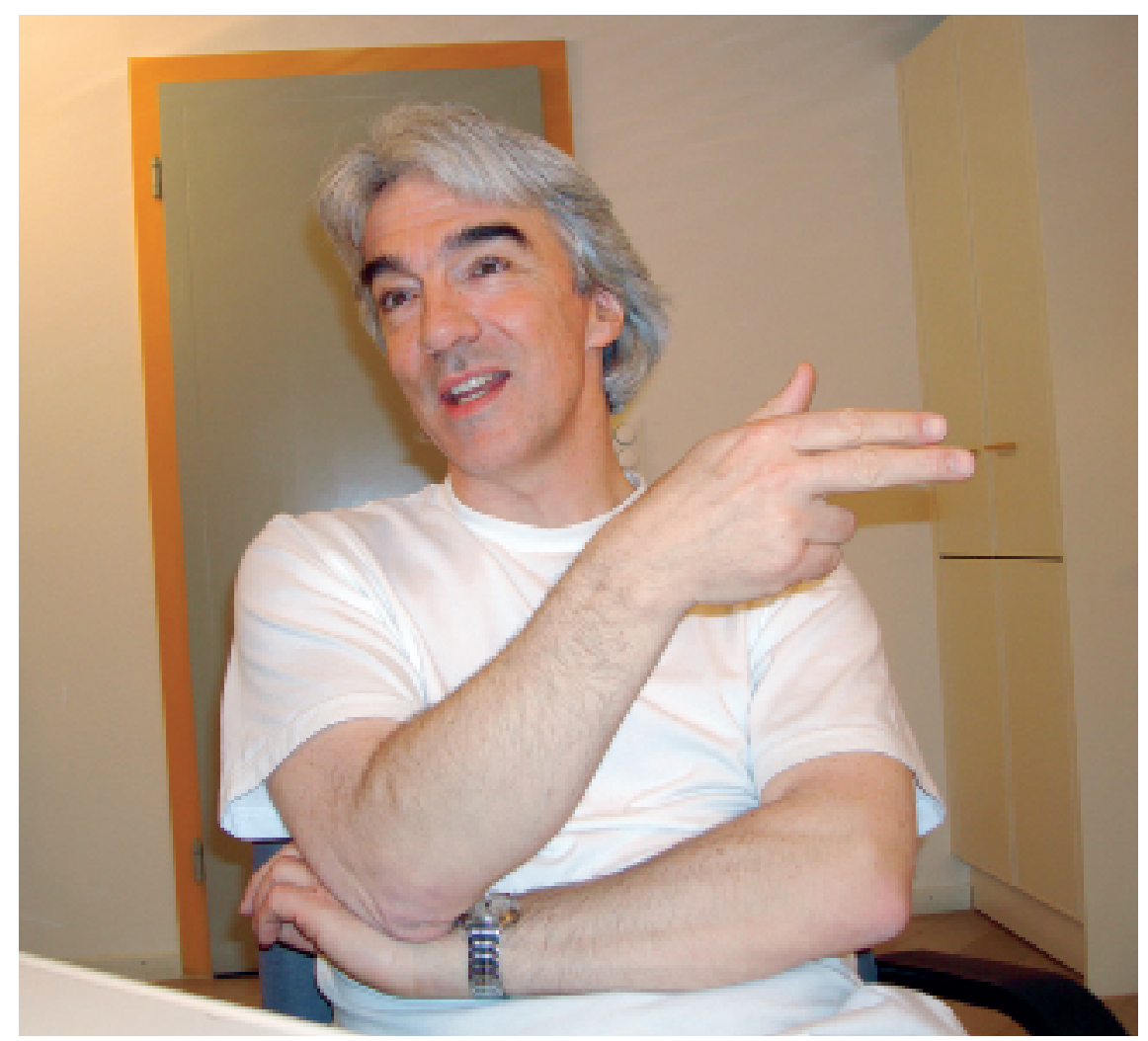

Späths Wunsch an die Politiker: «Es wäre schön, wenn ein echter Dialog in Gang käme.»

Kann man sagen, dass die Lobby der Hausärzte in den entscheidenden "Gremien der Macht» noch schwächer ist als die der übrigen Ärzteschaft? Ja, dem würde ich zustimmen. Der Grund liegt vor allem darin, dass wir universitär nicht verankert sind. Die grossen Lobbyisten, das sind die Professoren, die aufgrund ihrer Position viel Einfluss und Macht und Beziehungen im Hintergrund haben. Wenn ein Professor der Universität Zürich irgendetwas postuliert, dann hat das in der Politik, bei den Medien und damit auch in der Bevölkerung weitaus mehr Resonanz und Gewicht, als wenn der Hansueli Späth aus Langnau etwas sagt.
Wie erleben Sie persönlich als Hausarzt mit langjähriger Erfahrung die Verschlechterung der Arbeitsbedingungen?

Die administrative Belastung steigt. Die Unkosten steigen. Allgemein kann man sagen, die Arbeit wird immer weniger attraktiv, sie wird auch immer weniger lukrativ. Persönlich bin ich in der glücklichen Lage, nach 15 Jahren Hausarzttätigkeit gut etabliert zu sein. Ich habe meine Dignitäten. Es sind die Jungen, die leiden, die stark eingeschränkt werden. Die junge Generation hat diesen Panzer des Nicht-mehr-Dürfens und Nicht-mehr-Könnens, wenn es um das freie Praktizieren geht. Die spüren das viel mehr als wir Dinosaurier. Einzelpraxen wie meine werden Sie hoffentlich in zehn Jahren nicht mehr sehen. Das sind Auslaufmodelle, die keine Zukunft haben. Die Bedingungen dafür, dass die Jungen sich zusammenschliessen können, müssen aber erst noch verbessert werden.

\section{«Meine Vision ist, dass der 1. April der Tag der Hausarztmedizin wird, so wie es den Muttertag oder den Weltaidstag gibt.»}

So weit, so klar. Weshalb aber sollen Kolleginnen und Kolleginnen aus anderen Fachbereichen an die «Demo» gehen? Mit welchen Argumenten wollen Sie die nach Bern bringen?

Für mich wäre es ein ganz starkes Signal nach aussen, wenn sich alle Kolleginnen und Kollegen mit den - verstehen Sie diesen Ausdruck nicht falsch - «Schwächsten» der Ärzte solidarisieren würden. Das ist für mich die Zukunft, gesellschaftlich, politisch, sozial, auch standespolitisch. Nur wenn wir uns als Ärzteschaft untereinander solidarisieren und die Grabenkämpfe aufgeben, werden wir gemeinsam überleben können. Deshalb wünsche ich mir, dass sich alle Kolleginnen und Kollegen für die Hausärzte exponieren. Je mehr Ärzte aus verschiedenen Disziplinen kommen, desto stärker wird diese Botschaft wahrgenommen.

Was ist Ihr Wunschszenario, abgesehen von einer möglichst grossen Teilnehmerzahl? Soll der Anlass Volksfestcharakter haben?

Mein Wunsch wäre schon, dass es eine eindrückliche, friedliche Kundgebung wird. Ich hoffe, es werden einige Tausend Ärzte kommen. Kind und Kegel sollen dabei sein. Es ist ja eine Kundgebung für die Ärzte mit ihren Familien, den Praxisassistentinnen und wenn möglich mit den Pa- 
tienten. Wir wünschen uns viele fröhliche Gesichter. Nach der eigentlichen Demonstration wird es verschiedene Aktionen lokaler Gruppierungen geben. Auch eine Band wird für Stimmung sorgen, die «Hot Docs» aus Zürich. Es wird also sicher ein Fest geben. Mein Alptraum dagegen ist, dass ich alleine mit dem Vorstand der SGAM und hundert Nasen auf dem Bundesplatz stehe, und es regnet und schneit und hagelt...

Das wünschen wir Ihnen natürlich nicht. Angenommen, die Demonstration wird ein Erfolg: Was kommt danach?

Das ist wahrscheinlich das Allerwesentlichste: Der 1. April ist nicht Höhepunkt und Abschluss von irgendetwas, sondern soll den Beginn einer neuen Ära markieren, in der die Ärzte ihre Zukunft mitbestimmen können. Meine Vision ist, dass der 1. April der Tag der Hausarztmedizin wird, so wie es den Muttertag oder den Weltaidstag gibt. Und ich hoffe natürlich sehr, dass mit diesem ersten Event zum 1. April etwas bewirkt werden kann. Es wäre schön, wenn ein echter Dialog in Gang käme, wenn insbesondere die Politiker mit uns sprechen und die Hausarztmedizin in Zukunft wirklich unterstützen würden.

\section{Die Demonstration der Grundversorger} am 1. April

Vertreter der Hausärzteorganisationen werden am Vortag des 1. April in einer Medienkonferenz die Anliegen und Forderungen der Grundversorger bekanntgeben. Die offizielle Demonstration auf dem Bundesplatz in Bern beginnt um $14 \mathrm{Uhr}$ und dauert rund eine Stunde. Zur Teilnahme aufgerufen sind die Grundversorger mit ihren Familien und Patienten, die medizinischen Praxisassistentinnen, aber auch die gesamte Ärzteschaft und alle, die das Anliegen einer starken Hausarztmedizin unterstützen möchten. Im Rahmen der Kundgebung werden neben anderen Ständerätin Simonetta Sommaruga (SP) sowie FMH-Präsident Jacques de Haller sprechen. Im Anschluss an die Demonstration wird die Petition «Gegen die Schwächung der Hausarztmedizin und den drohenden Hausärztemangel» mit weit über 100000 Unterschriften im Bundeshaus deponiert werden. Ausserdem sind verschiedene Aktionen geplant, die dem Anlass Festcharakter geben sollen, beispielsweise ein Konzert der Ärzteband «Hot Docs». 